\title{
Effects of Non-Susceptible Hosts on the Infection with Trypanosoma cruzi of the Vector Triatoma infestans: an Experimental Model
}

\author{
Diego P Vázquez/+ , Delmi Canale*, Ricardo E Gürtler \\ Laboratorio de Ecología General, Facultad de Ciencias Exactas y Naturales, Universidad de Buenos Aires, \\ Ciudad Universitaria, 1428 Buenos Aires, Argentina *Servicio Nacional de Chagas, 9 de julio 356, \\ 5000 Córdoba, Argentina
}

\begin{abstract}
We tested experimentally the effects of the presence of non-susceptible hosts on the infection with Trypanosoma cruzi of the vector Triatoma infestans. The experiment consisted in two treatments: with chickens, including two chickens (non-susceptible hosts) and two infected guinea pigs (susceptible hosts), and without chickens, including only two infected guinea pigs. The hosts were held unrestrained in individual metal cages inside a closed tulle chamber. A total of 200 uninfected $\mathrm{T}$. infestans third instar nymphs were liberated in each replica, collected on day 14, and examined for infection and blood meal sources on day 32-36. The additional presence of chickens relative to infected guinea pigs: (a) significantly modified the spatial distribution of bugs; $(b)$ increased significantly the likelihoods of having a detectable blood meal on any host and molting to the next instar; (c) did not affect the bugs' probability of death by predation; and $(d)$ decreased significantly the overall percentage of $\mathrm{T}$. infestans infected with $\mathrm{T}$. cruzi. The bugs collected from inside or close to the guinea pigs' cages showed a higher infection rate (71-88\%) than those collected from the chickens' cages (22-32\%). Mixed blood meals on chickens and guinea pigs were detected in 12-21\% of bugs. Although the presence of chickens would decrease the overall percentage of infected bugs in short term experiments, the high rate of host change of T. infestans would make this difference fade out if longer exposure times had been provided.
\end{abstract}

Key words: Trypanosoma cruzi - Triatoma infestans - Triatominae - Chagas disease - infection - transmission zooprophylaxis - host-feeding patterns

The transmission of Trypanosoma cruzi, the etiologic agent of Chagas disease, is usually associated with impoverished rural areas in which houses built with local materials favor the development of vector populations. In Argentina and in other six countries in South America, the main vector is Triatoma infestans Klug (Hemiptera: Reduviidae) (Schofield 1985).

Dogs and cats are important host blood sources for domiciliary T. infestans (Wisnivesky-Colli 1987, Gürtler et al. 1996) and the main domestic reservoirs of T. cruzi (Minter 1976). In some areas of Bolivia and Perú, the domestic guinea pig (Cavia porcellus) is also an important reservoir of $T$. cruzi (Herrer 1955). In rural areas from northwestern

\footnotetext{
${ }^{+}$Corresponding author. Present address: Department of Ecology and Evolutionary Biology, University of Tennessee, Knoxville, TN 37996-1610, USA.

Fax: +1-423-974-3067. E-mail: vazquez@utk.edu Received 30 September 1998

Accepted 27 January 1999
}

Argentina, the proportion of domiciliary $T$. infestans infected with $T$. cruzi increased with the number or proportion of infected dogs or cats in the house (Gürtler et al. 1998).

Chickens are also important blood sources of domiciliary T. infestans (Wisnivesky-Colli 1987, Gürtler et al. 1996), but they are not susceptible to T. cruzi infection (Minter-Goedbloed \& Croon 1981). As stated by Minter (1976), the effect of the presence of chickens on transmission is not clear; it could be (1) beneficial, reducing the overall percentage of infected vectors (i. e. zooprophylaxis), or (2) detrimental, favoring the existence of high vector densities. Recently, Cecere et al. (1997), using multiple regression analysis, showed that the domiciliary density of $T$. infestans significantly increased both with the proportion of bugs that fed on chickens and with the householders' habit of letting hens nest in bedroom areas. Furthermore, the more T. infestans bugs fed on chickens or dogs the less they fed on humans (Gürtler et al. 1997).

The effects of non-susceptible hosts on parasite transmission have also been considered in other vector borne diseases. In some cases, the presence 
of non-susceptible hosts resulted in a reduced parasite transmission, while in other cases their presence led to an increased transmission rate (reviewed by Service 1991). To the best of our knowledge, no zooprophylaxis experiments have been published in relation with the transmission of $T$. cruzi. In this study, we look into the transmission pathway that goes from infected hosts to susceptible bugs, and present evidence of a reduced probability of infection of $T$. infestans with $T$. cruzi caused by the additional presence of chickens.

\section{MATERIALS AND METHODS}

Experimental design - Two matched replicas of the experiment were carried out at the Vector Reference Center of the Chagas National Service at Santa María de Punilla (Córdoba, Argentina). The mean daily temperature recorded during the experiments in January $\left(22.7^{\circ} \mathrm{C}\right)$ did not differ significantly from that recorded in March $\left(21.6^{\circ} \mathrm{C}\right)$ $(\mathrm{t}=1.289, \mathrm{~N}=14, \mathrm{P}>0.05)$. The experimental system was under the natural cycle of light-darkness.

The experiment was carried out in two chambers, each one consisting of a closed tulle tent of $2.8 \times 2.8 \mathrm{~m}^{2}$ and $1.4 \mathrm{~m}$ in height $\left(\sim 4 \mathrm{~m}^{3}\right)$ with an entrance in one of the corners. Each chamber was placed in a closed room to avoid direct exposure to rain or sun. Each host was placed alone in a metal cage on opposing corners. A pile of 32 adobe bricks provided refuge to the triatomines in the center of each chamber, on the assumption that the bugs would hide there and not in the metal cages. Each chamber was randomly assigned to one of two treatments: (1) with chickens, including two chickens and two guinea pigs infected with T. cruzi, and (2) without chickens, including only two guinea pigs infected with $T$. cruzi. The experiment was replicated twice, in January and March 1995.

A total of 200 uninfected T. infestans third instar nymphs was liberated on the adobe refuge in each replica. The nymphs were collected 14 days later, and stored in plastic vials at $27^{\circ} \mathrm{C}$ and $70 \%$ relative humidity for $18-22$ days to allow the development of $T$. cruzi infection. The guinea pigs were moved every four days among their respective cages in both treatments to minimize the effect of potential differences among subjects in their infectivity to bugs. In February, the guinea pigs were tested by xenodiagnosis to estimate their infectivity to the vector. A total of 10 uninfected third instar nymphs of $T$. infestans was applied on each guinea pig during $30 \mathrm{~min}$ and then inspected to verify that they had fully engorged. The bugs were individually examined for infection 30 and 60 days later as described below.

Animals - Ten-month old guinea pigs (Cavia porcellus) weighing about $700 \mathrm{~g}$ were used. The guinea pigs were in the chronic stage of infection, and had acquired $T$. cruzi infection (COB strain) through vectorial transmission four months before at the experimental field post of the Laboratory of Experimental Pathology (Universidad Nacional de Salta, Argentina). The same individuals were used in both replicas. Guinea pigs were chosen as susceptible hosts because (1) they are suitable hosts for T. infestans, (2) they have persistent parasitemia during the chronic stage of infection, and (3) they are easy to handle. Adult creole hens weighing approximately $1,800 \mathrm{~g}$ were used as non-susceptible hosts. Different hens were used in January and March.

Third instar T. infestans nymphs descending (F1) from adult $T$. infestans brought from natural populations were used for the experiments and xenodiagnoses. Nymphs were fed on chickens and reared at $27^{\circ} \mathrm{C}$ and $70 \%$ relative humidity. The nymphs' last feeding was as second instar nymphs, 30 days before starting the experiments or xenodiagnosis.

Laboratory methods - Fecal drops obtained by abdominal compression from each bug were diluted with $30 \mathrm{ml}$ of saline solution $(\mathrm{NaCl} 0.9 \%)$, covered with $22 \times 22 \mathrm{~mm}$ coverslips and thoroughly examined for active trypanosomes at $400 \mathrm{X}$. The number of microscopic fields necessary to find the first $T$. cruzi was taken as an index of the intensity of infection, assuming that the lower the density of $T$. cruzi the higher the number of fields needed to find at least one parasite.

All nymphs were dissected and the blood meals prepared and tested by agar double-diffusion as described by Gürtler et al. (1996). The antiserum against guinea pig serum was prepared in rabbits, as described by Gill (1984); it had a homologous titer of 1:4,000 and no cross-reactivity with chicken serum. The antiserum against chicken serum had a homologous titer of 1:1,600 and did not cross-react with guinea pig serum. Readings were made 24 and $48 \mathrm{hr}$ after filling the wells. A final reading was made after staining the dried agar plates with $0.1 \%$ amido black.

Although nymphs had been fasted for 30 days before the experiments, previous feeds on chickens were still detected in some cases. These precipitin bands, however, were much weaker and shorter than those from feeds made during the experiments. We therefore discarded short and weakly colored bands as evidence of a blood meal during the experiments.

Data analysis - The effects of the additional presence of chickens on attributes of the bugs (infection, molting, mortality, etc.) were described by a summary odds ratio (OR) and tested by the Mantel-Haenszel $\mathrm{C}^{2}$ test taking replicas carried out in 
January and March, or the bugs' collection site, as separate strata (Kahn \& Sempos 1989). The hostfeeding preference of bugs for chickens and guinea pigs was tested by the McNemar $\mathrm{c}^{2}$ test because the bugs were exposed simultaneously to both hosts (i. e. dependent events) within each replica with chickens.

\section{RESULTS}

Survival and molting - Of the 200 nymphs liberated at the beginning of each replica, from $70 \%$ to $76 \%$ were collected alive at the end of the experiments (Table I). The missing bugs were taken to have been preyed upon by the hosts. The presence of chickens exerted no significant effects on the probability of bug death beyond that attributable to the guinea pigs ( $\mathrm{OR}=0.87$; Mantel-Haenszel $\mathrm{c}^{2}=0.8, d f=1, \mathrm{P}>0.3$ ), but significantly increased the likelihood of molting to the next instar $\left(\mathrm{OR}=2.00\right.$; Mantel-Haenszel $c^{2}=17.5, d f=1$, $\mathrm{P}<0.001)$.

Host selection - Host selection by $T$. infestans was studied in two different ways: analysis of spatial distribution and analysis of host-feeding patterns. When the bugs from each hosts' cages were pooled - thereby forming three collection sites -, the spatial distribution of the bugs collected at the end of both experiments (Table I) differed signifi-

\section{TABLE I}

Number collected, spatial distribution and percentages of Triatoma infestans that moulted to fourth instar at the end of each experiment

\begin{tabular}{lccccc} 
& \multicolumn{2}{c}{ With chickens } & & \multicolumn{2}{c}{ Without chickens } \\
\cline { 2 - 3 } \cline { 5 - 6 } Collection place & January & March & & January & March \\
\hline Guinea pig's cage 1 & $46^{\mathrm{a}}$ & 18 & & 43 & 86 \\
Guinea pig's cage 2 & 30 & 38 & & 58 & 29 \\
Chicken's cage 1 & 11 & 33 & & - & - \\
Chicken's cage 2 & 17 & 18 & & - & - \\
Adobe refuge & 47 & 33 & & 49 & 37 \\
\hline Total & 151 & 140 & & 150 & 152 \\
& $(75)$ & $(70)$ & & $(75)$ & $(76)$ \\
\hline Percentage moulted & 44 & 66 & & 39 & 36 \\
\hline
\end{tabular}

$a$ : number $(\%)$ of insects collected. cantly between January and March $\left(c^{2}=11.8, d f=2\right.$, $\mathrm{P}<0.05)$. More bugs were collected from inside or close to the guinea pigs' cages than from inside or close to the chickens' cages in January, but bug counts were similar in March. The additional presence of chickens significantly decreased the likelihood of collecting nymphs from the guinea pigs' cages as compared to other sites when stratified by month $\left(\mathrm{OR}=0.33\right.$; Mantel-Haenszel $c^{2}=41.7$, $d f=1, \mathrm{P}<0.001)$.

Regarding host-feeding patterns (Table II), the presence of chickens significantly increased the odds of having a detectable blood meal on any host beyond that attributable to the guinea pigs $\left(\mathrm{OR}=2.25\right.$; Mantel-Haenszel $\mathrm{c}^{2}=16.7, d f=1$, $\mathrm{P}<0.001)$. In the presence of chickens, the proportion of bugs that fed exclusively on each host type did not differ significantly in January (McNemar $\mathrm{c}^{2}=0.4, d f=1, \mathrm{P}>0.1$ ), but significantly more bugs fed on chickens than on guinea pigs in March $\left(\mathrm{McNemar} \mathrm{c}^{2}=7.5, d f=1, \mathrm{P}<0.01\right)$. Mixed blood meals were detected in $12 \%-21 \%$ of nymphs. In the absence of chickens, 5 (4\%) bugs in January and 15 (10\%) bugs in March had blood meals on chickens, which indicates feeds prior to the experiments that were not eliminated by the selection procedure.

Bug infection - The percentage of T. cruzi-infected nymphs was significantly lower in the presence (range, 43-57\%) than in the absence of chickens (range, 68-92\%) when the effects due to month (January and March) and collection place (the guinea pigs' cages and elsewhere) were adjusted for by stratification using $42 \times 2$ tables $(\mathrm{OR}=0.32$; Mantel Haenszel $c^{2}=31.5, d f=1, \mathrm{P}<0.001$ ) (Table III). Both replicas showed similar qualitative effects of chickens on bug infection rates. Significantly more infected nymphs were detected in January $(74 \%, 224 / 301)$ than in March $(56 \%, 163 / 292)$ when stratified by the presence of chickens $\left(\mathrm{OR}=2.63\right.$, Mantel Haenszel $\mathrm{c}^{2}=26.8, d f=1$, $\mathrm{P}<0.001)$. When each replica was considered as a separate sampling unit, the number of bugs that were infected with $T$. cruzi after 30 days was linearly related to the number of bugs that fed on guinea pigs (Fig. 1).

TABLE II

Host-feeding sources of Triatoma infestans in January and March

\begin{tabular}{llcccrr}
\hline \multirow{2}{*}{ Replica } & Treatment & Guinea pigs & Chickens & Both & None & Total \\
\cline { 3 - 6 } January & With chickens & $41(27)$ & $48(32)$ & $32(21)$ & $30(20)$ & 151 \\
& Without chickens & $103(69)$ & $1(1)$ & $4(3)$ & $42(28)$ & 150 \\
March & With chickens & $38(27)$ & $67(48)$ & $17(12)$ & $18(13)$ & 140 \\
& Without chickens & $86(57)$ & $7(5)$ & $8(5)$ & $51(34)$ & 152 \\
\hline
\end{tabular}


TABLE III

Proportion of Triatoma infestans nymphs infected with Trypanosoma cruzi in each treatment

\begin{tabular}{lccccc}
\hline & \multicolumn{2}{c}{ January } & & \multicolumn{2}{c}{ March } \\
\cline { 2 - 3 } \cline { 5 - 6 } Collection place & With chickens & Without chickens & & With chickens & Without chickens \\
\hline Guinea pig's cages & $67 / 76^{a}(88)^{b}$ & $99 / 101(98)$ & & $40 / 56(71)$ & $88 / 115(77)$ \\
Chicken's cages & $9 / 28(32)$ & - & & $11 / 51(22)$ & - \\
Adobe refuge & $10 / 47(21)$ & $39 / 49(80)$ & & $9 / 33(27)$ & $15 / 37(41)$ \\
\hline Total & $86 / 151(57)$ & $138 / 150(92)$ & & $60 / 140(43)$ & $103 / 152(68)$ \\
\hline
\end{tabular}

$a$ : number of infected/number of examined insects; $b$ : percentage of infection.

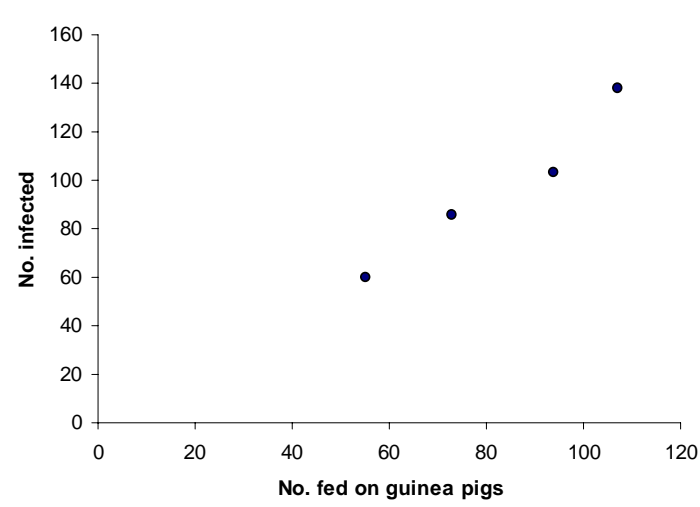

Fig. 1: linear regression of the number of infected nymphs by the number of Triatoma infestans nymphs with detected feeds on guinea pigs (including both those with detected feeds on guinea pig only and on guinea pig and chicken; $\left.\mathrm{P}<0.05, \mathrm{r}^{2}=0.95\right)$.

Within the treatment with chickens, infection rates were compared in relation to the bugs' collection place and host-blood source. The percentage of infected nymphs collected from the guinea pigs' cages was significantly greater than among those collected from the chickens' cages or the adobe refuge in both replicas $(\mathrm{OR}=12.62$, Mantel Haenszel $c^{2}=89.6, d f=1, \mathrm{P}<0.01$ ) (Table III). Nymphs that fed on guinea pigs only or on guinea pigs and chickens (range, 53-95\%) had a significantly greater infection rate than bugs that fed on chickens only (range, 13-15\%) when adjusted by month $\left(\mathrm{OR}=2.63\right.$; Mantel Haenszel $\mathrm{c}^{2}=26.8, d f=1$, $\mathrm{P}<0.001$ ) (Fig. 2). Bugs with non-detectable blood meals showed high percentages of infection both in the presence (range, 37-39\%, Fig. 2) and in the absence of chickens (range, 30-79\%, not shown).

The intensity of $T$. cruzi infection in the nymphs' feces did not differ significantly between treatments in both months by the Mann-Whitney test (January: $\mathrm{z}=0.72, \mathrm{P}>0.05$; March: $\mathrm{z}=0.86$, $\mathrm{P}<0.05)$. However, a significantly higher intensity of infection was recorded in January than in March either in the presence $(\mathrm{z}=2.26, \mathrm{P}<0.05)$ or in the

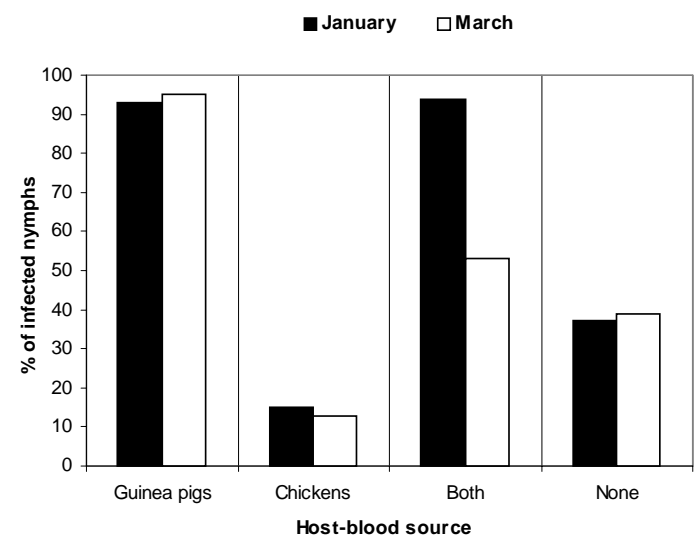

Fig. 2: proportion of Triatoma infestans nymphs infected with Trypanosoma cruzi in relation to host-feeding sources in the treatment with chickens.

absence of chickens $(\mathrm{z}=3.05, \mathrm{P}<0.01)$ (data not shown). On the average, the guinea pigs infected $80 \%$ of the xenodiagnosis nymphs. For each individual guinea pig, infectivity values were $60 \%$ (6/ $10), 70 \%$ (7/10), $90 \%$ (9/10) and 100\% (10/10).

\section{DISCUSSION}

Our study shows that the presence of chickens in addition to infected guinea pigs (a) modified the spatial distribution of bugs; (b) increased significantly the likelihood of having a detectable blood meal on any host and molting to the next instar; (c) did not affect the bugs' probability of death by predation; and (d) decreased significantly the overall percentage of $T$. infestans infected with $T$. cruzi.

Contrary to expectations supporting the use of mud bricks as a suitable refuge, most of the bugs chose to hide within or below the hosts' metal cages. This was a somewhat surprising finding in view of the substrate preferences of $T$. infestans (Wiesinger 1956), in which metal ranked far behind mud, and the very short distance between hosts and the adobe refuge. Our tentative conclusion is that host proximity dominated over substrate pref- 
erences under our experimental conditions. Host proximity has also been considered more important than host preference for triatomine bugs (Minter 1976).

Host availability affects the spatial distribution and nutritional status of $T$. infestans populations, and may therefore provide a density-dependent mechanism that leads to the regulation of population size (Schofield 1985). In accord with this general theory, our study shows that the likelihood of having a detectable blood meal on any host (a surrogate of bug nutritional status) and molting to the next instar in a specified time period increased significantly when two infected guinea pigs and two chickens hosts were available as compared with only two infected guinea pigs. Such differences most likely arose from an increase in bug feeding success and/or blood meal size per bug related to the availability of more hosts, or chickens, or both. The probability of bug disapearance was not significantly modified by host numbers or the additional presence of chickens. The additional presence of chickens most likely would boost up bug population size to a new carrying capacity.

The bugs preferred to feed on chickens rather than on the equally available guinea pigs, at least in one of the replicas. Although the chickens' biomass was approximately 2.6 higher than the guinea pigs', the excess number of bugs that fed on chickens relative to guinea pigs was well below any direct proportionality to host biomass. In other hematophagous insects, host density, size, attractiveness and tolerance to bites determined vector hostfeeding patterns (Edman et al. 1974, Edman \& Webber 1975, Lehane 1991). However, when different host species differed in their tolerance to bites and attractiveness, those relationships were less clear (Edman et al. 1974). In our study, both host species were initially equally accessible to bugs, but we have not assessed if they were equally attractive and tolerant to bites. As different chickens of similar size were used in January and March, the observed differences in host-feeding profiles between months may have been due to differences in the tolerance of individual chickens to bites, as observed by Rossel Reyes (1984).

Most of our current knowledge of triatomine host-feeding patterns come from blood meal identification studies of field samples (Minter 1976, Wisnivesky-Colli 1987, Gürtler et al. 1997), but this is not equivalent to host-feeding preferences (Washino \& Tempelis 1983). The host-feeding preferences of triatomine bugs have been studied experimentally only by Jirón and Zeledón (1982). In a simultaneous exposure of different vertebrate hosts to Rhodnius prolixus, $T$. dimidata and $T$. infestans, the bugs had no clear feeding preferences among dogs, chickens and opossums, but did not feed on toads; $T$. infestans, however, showed some preference for dogs in short day-time experiments, and for chickens in all-night trials (Jirón \& Zeledón 1982). The marked preference for warm-blooded hosts may be explained by the well known thermotropism of triatomine bugs (Núñez 1982). Whether thermotropism would explain the observed relative preference of $T$. infestans for chickens, which have a slightly higher body temperature than guinea pigs, remains to be determined. For a thorough understanding of triatomine host choices, host tolerance to bites and attractiveness would have to be considered.

The spatial distribution of bugs at the end of the experiments did not agree entirely with their host-feeding results, possibly because the spatial distribution results were an instantaneous representation of the system dynamics. As indicated by the proportion of mixed blood meals and the spatial distribution of the nymphs, there was a high rate of host change in both replicas. Furthermore, because our blood meal detection method does not allow distinguishing between blood meals on different individuals of the same host type, the effective rate of individual host change may have been even greater. This high rate of host change is characteristic of domiciliary T. infestans (WisniveskyColli 1987, Gürtler et al. 1996), although reasons for it remain unclear. In our system, host-feeding patterns provided a closer estimate of the host-vector contacts that determine bug infection with $T$. cruzi than the ultimate spatial distribution of bugs.

The presence of chickens significantly reduced the infection with $T$. cruzi of the vector T. infestans, in agreement with computer simulations (Himschoot 1993) and field results (Gürtler et al. 1998). This effect cannot be attributed to uncontrolled factors, such as differences in the actual degree of bug feeding between and within treatments. The proportions of nymphs that fed and molted in the presence of chickens were either similar or even greater than in the absence of chickens. Both in the insectary and the xenodiagnosis, over $95 \%$ of nymphs with a similar fasting period as in this study would normally feed when exposed to a restrained host for a few hours. In addition, the infectivity to bugs varied little among individual guinea pigs and most likely had no effect on the probability of bug infection as the guinea pigs were interchanged among cages regularly. It is noteworthy that $37-39 \%$ of bugs without a detectable feeding 18-22 days after finishing the trials were infected with $T$. cruzi, indicating that a very small blood meal on the infected guinea pigs was sufficient to produce a detectable infection. 
Chickens are insusceptible to $T$. cruzi due to the action of complement-mediated lysis (MinterGoedbloed \& Croon 1981). Urdaneta-Morales (1973) reported that additional feedings of infected $R$. prolixus on chickens, reptiles (also insusceptible to $T$. cruzi), mice or rabbits did not modify the proportion of $T$. cruzi infected bugs or its intensity of infection. The absence of effects of chicken blood on bugs with $T$. cruzi infection was also reported by several other authors (e.g., Minter 1976, p. 46). More recent results by Schaub (1988), however, showed that $T$. cruzi epimastigotes in the bug's stomach were clearly affected by a post-infective blood meal on chickens, but not on uninfected mice, both in vivo and in vitro. Although a biological interaction between $T$. cruzi and chicken blood components occurred in the bug's stomach, established intestinal infections of T. cruzi were not affected by the source of subsequent blood meals (Schaub 1988).

The overall prevalence and intensity of infection of the nymphs decreased substantially from January to March in spite of similar mean daily temperatures that fell within the range of optimum development for $T$. cruzi within triatomine bugs (Neves 1971). The differences between months might have originated from a decrease in the guinea pigs' parasitemia, but the percentage of nymphs that fed on guinea pigs and had a detectable T. cruzi infection was as high in March as in January. Differences between replicas therefore remain unexplained.

The proportion of infected nymphs differed between treatments mainly because of the lower prevalence of infection among bugs collected near the chickens or that fed on them. The presence of chickens had no effects on the prevalence of infection of bugs associated with the guinea pigs. Thus subgroups with different prevalence rates of infection coexisted within the vector population, as sometimes observed in rural houses (Rabinovich et al. 1990). Heterogeneity in the distribution of infection among vector and host populations is expected to augment the basic reproductive number $\left(R_{0}\right)$ as compared with homogeneously mixing populations (Hasibeder \& Dye 1988). Although in the short term the introduction of chickens would decrease the overall percentage of $T$. cruzi infected bugs in a given population, the observed high rate of host change would reduce such differences between treatments if longer exposure times had been used. More important, the introduction of chickens may boost up bug population size. In rural households of northwest Argentina, the presence of chickens exerted negative and significant effects on the proportion of infected T. infestans, as in the present study, but positive and significant effects on the number of domiciliary bugs (Cecere et al. 1997) and the number of $T$. cruzi-infected bugs collected per person-hour (Gürtler et al. 1998). This issue, however, is beyond the scope of our experimental model. In a more real scenario, increased bug densities or infected bug densities would likely result in an overall increase of parasite transmission rates to susceptible hosts, thereby excluding the use of chickens for zooprophilaxis.

\section{ACKNOWLEDGMENTS}

To Dr Abel Hurvitz (Servicio Nacional de Chagas, Argentina), Raúl Stariollo and staff at Santa María de Punilla for technical assistance. To Miguel Ángel Basombrío (Universidad Nacional de Salta) who kindly provided the infected guinea pigs. To Carla Cecere, Mónica Castañera, Rodrigo De Marco, Claudio Lazzari, Nicolás Schweigmann and two anonymous reviewers for comments; Juan Flo for guidance in the preparation of antisera, and Ana Haedo for statistical advice (Facultad de Ciencias Exactas y Naturales, Universidad de Buenos Aires).

\section{REFERENCES}

Cecere MC, Gürtler RE, Chuit R, Cohen JE 1997. Effects of chickens on the prevalence of infestation and population density of Triatoma infestans in rural houses of north-west Argentina. Med Vet Entomol 11: 383-388.

Edman JD, Webber LA 1975. Effect of vertebrate size and density on host-selection by caged Culex nigripalpus. Mosq News 35: 508-512.

Edman JD, Webber LA, Schmid AA 1974. Effect of host defences on the feeding pattern of Culex nigripalpus when offered a choice of blood sources. J Parasitol 60: 874-883.

Gill GS 1984. Production of antisera for the serological identification of blood-meals of arthropods. Trans $R$ Soc Trop Med Hyg 78: 233-234.

Gürtler RE, Cecere MC, Vázquez DP, Chuit R, Cohen JE 1996. Host-feeding patterns of domiciliary Triatoma infestans (Hemiptera: Reduviidae) in northwest Argentina: seasonal and instar variations. $J$ Med Entomol 33: 15-26.

Gürtler RE, Cohen JE, Cecere MC, Chuit R 1997. Shifting host choices of the vector of Chagas disease Triatoma infestans in relation to the availability of hosts in houses in north-west Argentina. J Appl Ecol 34: 699-715.

Gürtler RE, Cohen JE, Cecere MC, Lauricella MA, Chuit R, Segura EL 1998. Influence of humans and domestic animals on the household prevalence and density of Triatoma infestans infected with Trypanosoma cruzi in northwest Argentina. Am J Trop Med Hyg 58: 748-758.

Hasibeder G, Dye C 1988. Population dynamics of mosquito-borne disease: Persistence in a completely heterogeneous environment. Theor Pop Biol 33: 3153.

Herrer A 1955. Importancia del cobayo como reservorio de la enfermedad de Chagas en la región sudocciden- 
tal. Rev Med Exp 9: 45-55.

Himschoot PH 1993. Un Modelo de Simulación sobre la Dinámica Poblacional de Rhodnius prolixus y Triatoma infestans (Hemiptera, Reduviidae) y sobre la Dinámica de Transmisión del Trypanosoma cruzi. Comportamiento y Estabilidad, PhD Thesis, Facultad de Ciencias Exactas y Naturales, Universidad de Buenos Aires, 220 pp.

Jirón LF, Zeledón R 1982. Preferencias alimentarias de tres especies de Triatominae (Hemiptera: Reduviidae) en condiciones experimentales. Rev Biol Trop 30: 151-159.

Kahn HA, Sempos CT 1989. Statistical Methods in Epidemiology, Oxford University Press, New York, 292 pp.

Lehane MJ 1991. Biology of Blood-Sucking Insects, Harper Collins Academic, London, 288 pp.

Minter DM 1976. Effects on transmission to man of the presence of domestic animals in infested households, p. 330-337. In New Approaches in American Trypanosomiasis Research, Scientific Publication No. 318. Pan American Health Organization, Washington.

Minter-Goedbloed E, Croon JJAB 1981. The insusceptibility of chickens to Trypanosoma (Schizotrypanum) cruzi. Trans $R$ Soc Trop Med Hyg 75: 350-353.

Neves D 1971. Influencia da temperatura na evolução do Trypanosoma cruzi en triatominos. Rev Inst Med Trop 13: 155-161.

Núñez JA 1982. Food source orientation and activity in Rhodnius prolixus Stål (Hemiptera: Reduviidae). Bull Ent Res 72: 253-262.

Rabinovich JE, Wisnivesky-Colli C, Solarz ND, Gürtler
RE 1990. Probability of transmission of Chagas disease by Triatoma infestans (Hemiptera: Reduviidae) in an endemic area of Santiago del Estero, Argentina. Bull WHO 68: 737-746.

Rossel Reyes OJ 1984. A Comparison of the Feeding Performances of Rhodnius prolixus Stål and Rhodnius robustus Larrousse (Hemiptera, Triatominae), PhD Thesis, Imperial College of Science and Technology, London, 339 pp.

Service MW 1991. Agricultural development and arthropod-borne diseases: A review. Rev Saúde Públ 25: 165-78.

Schaub GA 1988. Direct transmission of Trypanosoma cruzi between vectors of Chagas' disease. Acta Trop 45: 11-19.

Schofield CJ 1985. Population dynamics and control of Triatoma infestans. Ann Soc Bélge Med Trop 65 (Suppl. 1): 149-164.

Urdaneta-Morales S 1973. Trypanosoma cruzi infections in Rhodnius prolixus refed on different hosts. Rev Inst Med Trop São Paulo 15: 218-221.

Washino RK, Tempelis CJ 1983. Mosquito host blood meal identification. Methodology and data analysis. Ann Rev Entomol 28: 179-201.

Wiesinger D 1956. Die Bedeutung der Umwelfaktoren für den Sugakt von Triatoma infestans. Acta Trop 13: 97-141.

Wisnivesky-Colli C 1987. Feeding patterns of Triatominae in relation to transmission of American Trypanosomiasis, p. 99-117. In RR Brener, AM Stoka (eds), Chagas Disease Vectors, Vol. 1, CRC Press, Boca Raton, Fl. 
Effects of Non-susceptible Host on Infection of $T$. infestans • Diego P Vázquez et al. 